\title{
Quality assurance in interventional cardiology
}

\author{
John Perrins, President, British Cardiovascular Intervention Society
}

The British Cardiovascular Intervention Society (BCIS) exists to aid the development and practice of percutaneous cardiac intervention in the UK. As such it has responsibilities to individuals who practise as well as responsibilities to their patients, departments, and hospitals. Quality assurance, peer review, and individual audit highlight the tensions between the society's responsibilities to individual practitioners and its wider responsibility to patients more sharply than any other activity of the society. However BCIS council strongly believes that proper quality assurance is the most effective way it has of supporting individual operators and departments throughout the UK.

BCIS began as a voluntary group of individual physicians who met to discuss case histories and issues relating to this rapidly growing field. As the speciality grew and matured so has the society, which now has several important functions.

(1) BCIS represents all interested parties, including physicians, technicians, nurses, and regulatory bodies, and has a very strong association with industry through the British Cardiovascular Industry Association.

(2) BCIS has an important role in teaching in its support and organisation of the yearly advanced angioplasty and regional autumn meetings, and by promoting many other educational activities throughout the year, of which this supplement is one.

(3) BCIS, together with the other affiliated groups, has formally joined with the British Cardiac Society such that BCIS has a representative sitting on the British Cardiac Society council. In addition, all guidelines produced by BCIS are now approved by the council of the British Cardiac Society before publication.

(4) Most importantly BCIS has a key role in determining the standards of care which interventionists and their teams should be providing for their patients. In 1996 a joint working group between the British Cardiac Society and BCIS set out guidelines for training and continuing competence in coronary angioplasty. ${ }^{1}$ This first document provoked much discussion and criticism but its general acceptance has defined BCIS's role in this area. Over the last two years, a second joint working group has updated the recommendations and outlined the requirements for optimal modern practice. This document, shortly to be published in Heart, not only covers training and continuing competence but also addresses the infrastructure required for good practice and highlights the importance of audit and peer review. ${ }^{2}$
For many years, BCIS has collected data on an annual basis that has been used for tracking the growth of the specialty. The early work of Peter Hubner and then Huon Gray encouraged all departments to submit voluntarily some data to BCIS. The data were largely related to procedure volumes, centre numbers, etc. Attempts were made to determine clinical outcomes after coronary intervention, but it became apparent that there were many flaws. The current public and political interest in outcomes after medical interventions will undoubtedly accelerate the process that BCIS has been advocating for many years. It is no good just providing good clinical practice; we have got to be seen to provide good clinical practice. For the determination of standards against which activity can be monitored, the society believes in a process run by the profession for the profession and believes that self regulation is the only methodology suitable for this process. The concept of non-medical regulation of standards would not be acceptable to most clinicians. The key issues then are the processes of audit and peer review, and central to these is the requirement for data collection.

\section{Audit and peer review}

AUDIT SYSTEM

Audit should determine whether we are doing something we should be doing (appropriateness) and whether we are doing it well (quality). If deficiencies are found, then solutions should be sought, implemented, and their effect monitored. To allow an audit process to develop, it was apparent some years ago that BCIS would need to modify its data collection system. At that time, rather than progress unilaterally, BCIS entered into a collaborative project with other professional bodies in the field of cardiovascular medicine. These included the Society of Cardiothoracic Surgeons, the Heart Valve Registry, the Association of Cardiothoracic Anaesthetists, the British Pacing and Electrophysiology Group, and more recently those representing the field of paediatric cardiology.

In discussion with the Department of Health, the concept of the central cardiac audit database (CCAD) was developed. ${ }^{3}$ Over the last few years a pilot project involving five cardiothoracic centres in the UK has been running. The remit of this project was to determine whether it is possible to collect a comprehensive set of data on individual patients and transmit it electronically to a central database. The pilot project is nearing completion and we await a decision from the Department of Health as to whether this methodology should be used by all UK cardiothoracic centres. This may well come under the jurisdiction of the National Institute for Clinical Excellence. It is 
not clear to the society at this stage whether there will be a mandate to use CCAD or whether a modified form of regulation is to be used. We can take it for granted, however, that "roll out" in some form or other is shortly to occur. Regardless of the Department of Health's intentions, BCIS strongly recommends that the process of data collection developed by the CCAD project is implemented in all interventional centres.

In the CCAD pilot project, each of the societies developed a group whose remit was to provide a minimum dataset for their respective specialities. The BCIS audit subcommittee has determined the fields to be used for coronary intervention. These have been approved by the BCIS council. They include a core dataset that is an absolute minimum, but BCIS is also interested in collecting more data and it is recommended that departments also collect the additional dataset. Without these data, case mix analysis and risk stratification will be impossible. At present, BCIS has concentrated on coronary interventions, but this exercise will be broadened in future to cover other areas of cardiac intervention (valvuloplasty, hole closures, septal vessel ablation, etc). Whether we use CCAD or some other central data collection exercise is yet to be determined but it is essential that we collect accurate data. In collaboration with the Society of Cardiothoracic Surgeons in particular, we are strongly of the view that each part of this process has individual responsibilities.

(1) Hospital trusts must provide the infrastructure required for prospective patient orientated data collection. This includes the necessary hardware and software but also the data collection personnel and the space within cardiac departments to develop a comprehensive data collection system. The trust will also appoint somebody to be responsible for clinical governance who will have the right to ask individual operators about their case mix and clinical outcomes.

(2) The cardiac department should appoint a key clinician to lead the audit process and should determine the method for data collection, verification, collation, and transmission to BCIS (or, in future, the CCAD). Clearly the data are required for an internal review process, but transmission to a central database is required to allow for a comparison with overall national activity. Each department should have regular audit meetings for interventional cardiology.

(3) The individual operator should assume full responsibility for collection of clear, accurate, and honest data on all cases under his or her care, provided the trust and the cardiac department have provided the infrastructure to do this. The individual will also be expected to provide outcome data to the lead clinician within the trust responsible for this area of clinical governance.

(4) BCIS will provide the minimum dataset, define variables and, in conjunction with the other societies using the CCAD, develop and provide statistical models enabling risk analysis and comparison of outcomes. BCIS council will also develop a method of external data validation and peer review. In addition it will provide a source of contact for professional guidance.

(5) The Royal College of Physicians specialist advisory committee for higher training will ensure that effective audit meetings occur within departments and that appropriate levels of staff and expertise are available for training of juniors.

As part of clinical governance, each trust will ask individual units and operators to review the results of their clinical activity and monitor it against some form of bench mark. For coronary intervention, this will not be possible without the CCAD or an equivalent system.

As proposed, the CCAD will be an anonymous process. Each unit will have a code. Each operator within a unit will have a code that will obviously be known to the key individual within a department but not identifiable by the CCAD. Each operator will have a key into the database. This key will allow that individual to analyse his or her results, and will also allow access to aggregated national data. The proposed reporting system will also allow that individual access to the aggregated results for his or her unit. The case mix of the individual will also be identified, as will the aggregated case mix for the unit and the UK.

BCIS has requested the following reports to be made available from the CCAD:

(a) case mix (by clinical syndrome)

(b) hospital outcome defined as major adverse cardiac events (MACE) and freedom from MACE analysed in relation to the clinical syndrome of the patient

(c) hospital outcome defined as MACE and freedom from MACE for the following index case-first time, single vessel, single lesion angioplasty in an elective patient in a non-occluded vessel.

In due course, it is hoped that follow up data at 30 days, one year and five years will be analysed, but at this stage BCIS requests hospital discharge data alone.

At present, the society would only have access to the national data but would not be able to identify individual departments or operators. The audit officer would compile a yearly report from these data without knowledge of departments or individuals. With this latter system, the maintenance of anonymity until the level of the trust's lead clinician would allay many fears that have been expressed. However, there are grounds for proposing an alternative system.

It is likely that the individual responsible for clinical governance within a trust will delegate responsibility to the lead clinician within an individual department. Individual operators will have to report the results of their activity to the lead clinician. In reality, it is likely that operators would give their permission for a lead clinician within their department to access the CCAD on their behalf. Thus, at the central 
level, the anonymity of the process continues, but anonymity is broken down at departmental level (as at present). This allows each department to have an internal review process in which results can be monitored against departmental and national activity. However, each trust may have a different view of this process and it is possible that some trusts will perform this process at managerial level, rather than at a clinical level. Many clinicians may find this unacceptable.

BCIS could have a key role in this process. One option is for each individual department (and thus each operator) to sign up to a society led process. Lead clinicians within each department would give permission to the audit officer of the society to access the database on their behalf and to compile a yearly report giving a breakdown of data at national, departmental, and possibly individual operator level (but still in an anonymous format). This would then be fed back to each department. BCIS council believes that this proposal allows for a process of external peer review but accepts that this can only be done with the permission of each individual operator and department. With such a system, the audit officer would know the individual results of each department, but this is as it is with the present system of reporting. The audit officer has, however, always maintained strict confidentiality.

Were this system to be developed into a process of external peer review, a review body would be required to assess the data. BCIS council proposes the development of a standards monitoring committee. This would consist of the president, the president elect, and the audit officer of the society. Individual centres would be compared with national activity. Analysis would take into account case mix and, in future, a risk stratification process. At present the CCAD project board is taking advice from professional biomedical statisticians about appropriate and robust methods of analysing and comparing clinical results. It is likely that methods will demand an analysis of trends over several years' activity before meaningful conclusions can be made. With the aid of such methods, the standards monitoring committee would be able to report back to BCIS council and be responsible for feedback to individual centres. Any published yearly reports would only reflect national activity.

In certain circumstances, it is conceivable that this committee could raise concerns about a centre's results. The standards monitoring committee would, in the first instance, raise those concerns with the lead audit clinician of the relevant department and if necessary with the relevant trust. If a request for help from a trust was received by BCIS either in response to a query from BCIS or as a result of a locally generated concern then a rapid response team would be formed. The concept of a rapid response team has already caused some concern among members of the society. It should be seen, however, as part of the process of self regulation and has been adopted or is being considered by other groups, including the Society of Cardiothoracic Surgeons.
This body would be set up by the president, on behalf of council, in response to a request from a trust. It would consist of the president of BCIS, a senior member of the British Cardiac Society, and an acknowledged expert in the relevant field. The team would arrange to visit the relevant department and produce a report that would be discussed with the relevant trust. If this process did not result in resolution of the issues then at the time of writing no further action could be taken by BCIS as it has no remit or authority to do so; the final responsibility for clinical governance lies with each trust. However the British Cardiac Society is currently considering the issue of peer review in a wider context and it may be that other mechanisms to interact with the trusts will become available in the future. The rapid response team would therefore be just a quick acting version of a regular peer review visit.

PEER REVIEW SYSTEM

In response to a request by the British Cardiac Society, BCIS is developing a methodology for a 3-5 yearly external review process (similar to the process used by the Royal College of Physicians in accrediting departments as training centres). Indeed, approval from the Royal College of Physicians is being sought. This will include a visit from a team of senior interventionists who will report on the standards set by individual departments. The make up of the team is yet to be determined, but one proposition is that the team should consist of a current member of council, an interventionist from the department that was reviewed last, and an interventionist from the centre that will be reviewed next. The visits would include an assessment of equipment and infrastructure, weekly timetables and rotas, staffing levels, and participation in the audit process. The team would report back to individual trusts as well as to BCIS council. The standards against which departments would be measured would be taken from the joint societies' working group guidelines which are to be published soon. BCIS has initiated a pilot project to look at the feasibility of such a process and the resources required, and will later report to the British Cardiac Society. This should not be seen as a threat but as a very positive process. First, good practice in one department could be recognised and taken up by other units. Secondly, those departments that have to practise interventional cardiology with inadequate facilities or support would be helped, as the reports from the society would be an impetus to trusts to develop a more appropriate infrastructure.

\section{The way ahead}

Each of the societies participating in the CCAD pilot project would like to see this process implemented at a national level. We await the deliberations of the Department of Health. Each department should, however, prepare itself for change. Over the last few years, BCIS has continued to collect what data it could, and in this supplement the results of the returns for 1992 to 1996 are presented. ${ }^{4}$ The 1997 returns are also included but the 
data are analysed in a way which highlights the problems we have encountered. A number of key recommendations are made. ${ }^{5}$ BCIS strongly recommends that each department practising interventional cardiology either modifies its database to become compatible with the minimum dataset or, if it has not already, develops a computer based database. BCIS does not make any recommendations about the software to be used as there are many programs that can do the job, some commercially available and some that have been developed within departments. Accurate and honest data collection and transmission is essential. Departments must employ key personnel to allow this to happen and give them time and space to fulfil their roles.

If a central database is to be used, CCAD is the preferred option. Centres will therefore have to be compatible with the central system. A definition of compatibility will be sent out to all departments once further information is received from the Department of Health. BCIS believes that this infrastructure will allow professional self regulation within the interventional community and provide a mechanism for openness and a fair evaluation of clinical standards. The old voluntary and paper based methods used by BCIS will not suffice. The proposed system should allay any individual fears about inappropriate reporting of activity and should be a tool to encourage purchasing bodies and trusts alike to provide the appropriate infrastructure required by interventionists to provide best practice. It provides a mechanism of professional support and guidance to allow us all to achieve the best possible clinical standards for our patients. The current BCIS council is of the view that we have to provide a fair and open system of self regulation and that we should avoid the possibility of having an unfair process foisted on us from outside. Whether we develop the audit process as one that encourages internal review only, or one which incorporates external review, will depend on further discussion within the society. The present council is of the view that an external process both for audit and assessment of infrastructure would be far more robust and that individual departments and operators would then be protected from any unfair accusation of hiding results. It would also provide the mechanism of ensuring that trusts provide an appropriate working environment. Given all of this, departments would be at a significant disadvantage if they were not seen to collaborate with this UK audit and peer review process.

Finally, it should be understood that standards of clinical practice, peer review, quality assurance, and clinical governance are the same wherever percutaneous intervention is practised in the UK. The private sector is in many ways even less well regulated than the National Health Service but individual operators cannot assume that these principles only apply to the health service sector. It is in my view mandatory that all centres of whichever complexion participate in this process fully and openly.

1 Parker DJ, Gray HH, Balcon R, et al. Planning for coronary angioplasty: guidelines for training and continuing competence. Heart 1996;75:419-25.

2 Gray HH, Swanton RH, Schofield PM, et al. Coronary angioplasty: guidelines for good practice and training. Recommendations of the joint working group on coronary angioplasty of the British Cardiac Society (BCS) and the British Cardiovascular Intervention Society (BCIS). Heart. [In press.]

3 Rickards A, Cunningham D. From quantity to quality: the central cardiac audit database project. Heart 1999;82 (suppl II):II1 $18-22$.

4 Gray HH, on behalf of the Council of the British Cardiovascular Intervention Society. Cardiac interventional procedures in the United Kingdom 1992-1996. Heart 1999; 82(suppl II):II10-17.

5 de Belder MA, on behalf of the Council of the British Cardiovascular Intervention Society. Cardiac intervention procedures in the United Kingdom 1997. Developments in data collection. Heart 1999;82 (suppl II):II2-9. 